\title{
THE INITIAL COMPOSITION AS AN ADDITIONAL PARAMETER DETERMINING THE MELTING BEHAVIOUR OF NANOPARTICLES (A CASE STUDY ON Si -Ge $_{1-x}$ ALLOYS)
}

\author{
Shishulin A.V. ${ }^{{ }^{*}}$, Potapov A.A. ${ }^{2,3}$, Shishulina A.V. ${ }^{4,5}$ \\ ${ }^{1}$ G.A. Razuvaev institute of organometallic chemistry, Russian Academy of sciences, Nizhny Novgorod, Russia, \\ chichouline_alex@live.ru \\ 2V.A. Kotel'nikov Institute of Radio Engineering and Electronics, Russian Academy of Sciences, Moscow, Russia \\ 3JNU-IREE RAS Joint Laboratory of Information Technology and Fractal Processing of Signals, Jinan University, \\ Guangzhou, China \\ ${ }^{4}$ R.E. Alekseev Nizhny Novgorod State technical University, Nizhny Novgorod, Russia \\ ${ }^{5}$ N.I. Lobachevsky Nizhny Novgorod State University, Nizhny Novgorod, Russia
}

\begin{abstract}
Being the basis of modern electronics, silicon-germanium semiconductor alloys are widely used in a great number of fields. In this paper, the phase equilibria in Si-Ge nanoparticles have been simulated using a thermodynamical approach. Calculations show that nanoparticles of different diameters and compositions have unique sets of nanoscale liquidus and solidus temperatures which differ significantly from the reference data for the bulk alloy, and the range between nanoscale liquidus and nanoscale solidus temperatures narrows with reducing the particle size. Unlike bulk alloys, the compositions of co-existing liquid and solid phases at different temperatures dramatically differ in nanoparticles with various Si contents while the dependences of equilibrium phase compositions and volume fractions of co-existing phases on particle diameters have turned out to be different at various temperatures and atomic fractions of Si in a nanoparticle. A thermo-dynamical interpretation of the obtained results has also been given based on several mechanisms of lowering the free energy of the system.
\end{abstract}

Keywords: phase equilibria, nanoparticles, melting, silicon, germanium.

\section{Introduction}

Investigation of solid solutions between silicon and germanium at the nanoscale has been of particular increasing interest in recent years. Si-Ge solutions possess a unique set of properties including wide band gap, which can be gradually changed (by varying the germanium content); high mechanical strength; resistance to high temperatures etc [1]. Those properties, some of which can be changed and improved by nanostructuring, make Si-Ge solutions be a very promising material in numerous fields.

Silicon is considered to be the basis of modern electronics, being a relatively inexpensive and abundant semiconductor. Silicon-germanium nanoalloys have been applied to designing metal-oxide-semiconductor field-effect transistors with improved value of carrier mobility, complementary metal-oxidesemiconductor integrated circuits, high speed and high responsivity waveguide photo-detectors and heterojunction bipolar transistors with dramatically improved performance, including values of cut-off frequency approaching to $300 \mathrm{GHz}$, and values of gate delay well below $10 \mathrm{ps}$ (the numbers unimaginable in bipolar transistors), add to this, excellent electronic and optical properties allow Si-Ge nanoalloys to be widely utilized in phase change memory (PCM) devices, directly composing some of the PCMs (see [2-5]).

Furthermore, Si-Ge alloys have been widely used for manufacturing thermoelectric generators of different types. Radioisotope $\mathrm{Si}-\mathrm{Ge}$ thermoelectric generators are installed on various spacecraft (e.g. "Voyager 2") and are working in space for several years almost without deterioration [6]. Thermoelectrical efficiency, which is revealed by a dimensionless figure of merit, $Z T$, can be greatly enhanced through nanostructuctural engineering [7, 8]. ZT is directly proportional to the electrical conductivity and inversely proportional to the thermal conductivity; the main approach to the solution of the problem of an increase in $Z T$ is using polycrystalline nanomaterials where the thermal conductivity is decreased due to the formation of multiple grain boundaries which scatter thermal phonons while retaining the values of other parameters, and some other effects (see [8] and Refs. within). 
Various types of Si-Ge nanostructures are prepared mainly by melt growth techniques including the zone-melting growth or the vapor-liquid-solid growth technology, however, the key role in fabrication of polycrystalline nanoalloys now belongs to additive manufacturing including direct and selective laser melting [9]. To control and improve those processes, the knowledge of thermodynamic properties at high temperatures (i.e. the phase diagram of Si-Ge solutions) at the nanoscale is critically needed. In the bulk state, pure Si and Ge and their alloys have been fairly well studied [10-12]. In nanosystems, experimental investigations (calorimetry experiments) are extremely challenging and time-consuming to perform, but nanothermodynamical calculations offer a smart alternative. Despite the fact that some attempts to theoretically predict the phase equilibria in Si-Ge nanoalloys have been made by L.H. Liang et al. [13], S. Bajaj et al. [14] or by B. Bonham and G. Guisbiers [15] and, there are still a lot of gaps in their description and the considered alloys remain poorly studied at the nanoscale.

In nanomaterials, the description of phase transformations and equilibria is significantly complicated due to some specific effects. The compositions of co-existing phases and phase transition temperatures in nanoscale systems (nanoparticles, nanodroplets etc.) depend on the size of the system [13-23], its shape [16$19,22]$ as well as on the thermodynamical properties of all interfaces [23]. In phase diagrams, the mentioned effects can manifest themselves as significant shifts of characteristic points, shifts and deformations of characteristic lines in comparison with phase diagrams for the bulk-state systems. The need to recalculate the phase equilibria at the nanoscale is due to the fact that the displacements of characteristic lines and points for nanoscale systems can exceed hundreds of degrees [13]. These effects have been observed experimentally [24] and can be explained by a great enhancement of the surface-to-volume ratio and surface contribution to the total energy of the system.

In order to calculate the phase equilibria at the nanoscale by thermodynamical methods, it is necessary to minimize the total Gibbs function of the system with allowance for the contribution of all interfaces and to analyze the configuration of its minima (see [16,20,21], for example). Despite the fact that there is a set of papers observing the mentioned effects, the general regularities cannot be obtained from most of them at present due to the substantial differences in the models derived and some critical gaps. For example, as far as the authors are aware, most of the approaches lack the consideration of the dependence of phase diagrams on the composition of the system. The model with the composition effect taken into account (with some simplifying presuppositions) has been introduced by our group and applied to modeling the melting behavior of nanoparticles of a tungsten heavy alloy for special applications [25] as well as the liquid-liquid phase separation in organic and polymeric solutions in small-volume droplets and pores [26]. The calculations have shown that, unlike bulk alloys, where a variation of the chemical composition of the system leads to changes in the volume fraction of co-existing phases (according to the lever rule) but does not affect the mutual solubility of components, in small-volume systems the chemical composition of the system determines not only the volume fractions of the phases, but also their equilibrium composition. Also, the calculations have shown an unusual effect of the significant variation of all phase equilibria characteristics depending on mutual position of co-existing solid and liquid phases in the structure $[8,16,19,20,23]$ while all the sets of characteristics dramatically differ from each other and from the corresponding values for the bulk state. The shape of the system can also be taken into account using different approaches. For example, in $[8,16,17$, 19], we have suggested a dimensionless coefficient of shape, which is equal to the ratio between the surface areas of the figure under consideration and the sphere of the same volume.

The shape coefficient is a continuous parameter generalizing various versions of isochoric transformations of the system, including smooth deformations. Another way to describe the complex morphology of real particles is using the methods of fractal geometry [8, 16-19]. Formation of highly complicated fractal-shaped structures is generic for many non-equilibrium processes [27], the morphology of such structures is characterized by their fractal dimensions: the examples of structures with various fractal dimensions can be found in [8, 17-19].

In the present paper, we have highly developed the approach from [25] and used it to predict the high temperature phase equilibria for Si-Ge solid core-liquid shell nanoparticles as well as the tendencies which characterize their deviations from the data for the bulk state. In the bulk state, the considered alloy has a lowtemperature symmetric miscibility gap at temperatures below $266.5 \mathrm{~K}$ [14]. Above this temperature up to the liquidus, the Si-Ge system exhibits continuous solubility in the solid state with a diamond structure. At high temperatures, Si-Ge alloys have liquidus and solidus curves without a congruent melting point. 


\section{A mathematical model of solid $\rightarrow$ liquid phase transitions in nanoscale particles}

The system to be modeled has the form of spherical Si-Ge nanoparticles of various diameters and compositions at temperatures above solidus. It is assumed that in phase equilibrium a nanoparticle is consisted of a single spherical solid inclusion (core-phase) surrounded by a spherical layer of a melt (shellphase). In a closed binary thermodynamic system, the conservation conditions of matter are given by Eqs. (1):

$$
\frac{\pi d^{3}}{6}=\sum_{j} V_{j}, n_{1}=x n, n_{2}+n_{1}=n, n_{i}=\sum_{j} n_{i j}, V_{j}=\sum_{i} n_{i j} V_{i}, x_{i j}=\frac{n_{i j}}{\sum_{i} n_{i j}}
$$

where $x$ is the atomic fraction of $\mathrm{Si}$ in the whole particle; $d$ is the diameter of the particle under consideration; $n$ is the total number of moles of both components in the particle; $V_{j}$ is the volume of phase $j$; $j=c, s$; the indices $c$ and $s$ refer to the solid core- and liquid shell-phases, respectively; $n_{i}, n_{i j}$ and $V_{i j}(T)$ are the number of moles of each component $n_{i}$, the number of moles of component $i$ in phase $j$ and the molar volume of component $i$ in phase $j$ (the molar volumes are considered to be temperature-dependent in general case), respectively; $i=1,2$; the indices 1 and 2 refer to $\mathrm{Si}$ and Ge, respectively; $x_{i j}$ is the molar of component $i$ in phase $j$.

Temperature-dependent molar volumes of pure solid Si and Ge (thermal expansion) are expressed as follows: $V_{1 c}=11.989 \cdot \exp \left(2.280 \cdot 10^{-5} \cdot(T-293)\right), V_{2 c}=13.648 \cdot \exp \left(1.725 \cdot 10^{-5} \cdot(T-293)\right)$ [28]. For melts of pure components: $V_{1 s}=11.100 \mathrm{~cm}^{3} / \mathrm{mol}, V_{2 s}=12.966 \mathrm{~cm}^{3} / \mathrm{mol}$ [ibid]. The geometric characteristics of the forming phases can be described as follows: $A_{c}=4 \pi\left(3 V_{c} / 4 \pi\right)^{2 / 3}, A_{s}=\pi d^{2}$ where $A_{c}$ are $A_{s}$ are the surface areas of the core- and shell-phases, respectively.

A criterion for whether the system is at equilibrium is a minimum of its Gibbs function with allowance of all interfaces: $g=\left(n_{1 c}+n_{2 c}\right) G_{c}\left(x_{1 c}, T\right)+\left(n_{1 s}+n_{1 s}\right) G_{s}\left(x_{1 s}, T\right)+\sigma_{s} A_{s}+\sigma_{c s} A_{c}$ where $\sigma_{s}, \sigma_{c s}$ are the surface energies on the outer (-shell) and internal (core-shell) boundaries. $G_{c}\left(x_{1 c}, T\right)$ and $G_{s}\left(x_{1 s}, T\right)$ are the molar Gibbs functions of solid and liquid Si-Ge solutions with compositions $x_{1 j}$ at temperature $T$, respectively.

As the authors of [10] have shown, the Si-Ge system can be described by the model of a regular solid solution:

$$
\begin{aligned}
& G_{s}\left(x_{1 s}, T\right)=R T\left(x_{1 s} \ln x_{1 s}+\left(1-x_{1 s}\right) \ln \left(1-x_{1 s}\right)\right)+\Omega_{s}\left(r_{s}\right) x_{1 s}\left(1-x_{1 s}\right), \\
& G_{c}\left(x_{1 c}, T\right)=R T\left(x_{1 c} \ln x_{1 c}+\left(1-x_{1 c}\right) \ln \left(1-x_{1 c}\right)\right)+\Omega_{c}\left(r_{c}\right) x_{1 c}\left(1-x_{1 c}\right)+ \\
& +x_{1 c} \frac{\Delta_{m} H_{1}\left(r_{c}\right)}{T_{m 1}\left(r_{c}\right)}\left(T-T_{m 1}\left(r_{c}\right)\right)+\left(1-x_{1 c}\right) \frac{\Delta_{m} H_{2}\left(r_{c}\right)}{T_{m 2}\left(r_{c}\right)}\left(T-T_{m 2}\left(r_{c}\right)\right)
\end{aligned}
$$

where $R$ is the ideal gas constant; $\Omega_{f}\left(r_{f}\right)$ are the regular solid solution parameters for liquid and solid phases, each parameter depends on the characteristic size $r_{j}$ of the considered phase (on the radius of the solid core-phase $r_{c}$ and the thickness of the liquid layer which forms the shell-phase $r_{s}$; both $r_{c}$ and $r_{s}$ can be obtained from the given Eqs. after some simple transformations); $\Delta_{m} H_{i}\left(r_{c}\right)$ and $T_{m i}\left(r_{c}\right)$ are the sizedependent melting enthalpies and melting points of pure $\mathrm{Si}$ and $\mathrm{Ge}$.

Size-dependent regular solution parameters $\Omega_{f}\left(r_{f}\right)$, melting enthalpies $\Delta_{m} H_{i}\left(r_{c}\right)$ and melting points $T_{m i}\left(r_{c}\right)$ as well as melting entropies $\Delta_{m} S_{i}\left(r_{c}\right)=\Delta_{m} H_{i}\left(r_{c}\right) / T_{m i}\left(r_{c}\right)$ can be calculated within various approaches; in this report, we use simple equations obtained by L.H. Liang et al. $[13,18]$ :

$$
\Delta_{m} S_{i}\left(r_{c}\right)=\Delta_{m} S_{i \infty}\left(1-\left(\frac{r_{c}}{a_{i}}-1\right)^{-1}\right), T_{m i}\left(r_{c}\right)=T_{m i \infty} \exp \left(\frac{-2 S_{i \infty}}{3 R\left(r_{c} / a_{i}-1\right)}\right), \Omega_{j}\left(r_{j}\right)=\Omega_{j \infty}\left(1-\frac{2 a_{i}}{r_{j}}\right)
$$

where $S_{i \infty}, T_{\text {mix }}$ are the "literature" melting entropies and melting points for pure $\mathrm{Si}$ and Ge in the bulk state; $\Omega_{f \infty}$ are the regular solid solution parameters for solid and liquid phases in the bulk state; $a_{i}$ is the characteristic size of the crystal structure of a pure component; in calculations below, $a_{i}$ is considered to be 
equal to 6 atomic radii [13]. According to a remark made in [13], we use in our calculations the atomic radius of $\mathrm{Si}$ which is higher than the one of $\mathrm{Ge}$ For pure $\mathrm{Si}$ : $T_{m 1 \infty}=1693 \mathrm{~K}, \quad \Delta_{m} S_{1 \infty}=29.41 \mathrm{~J} /(\mathrm{mol} \cdot \mathrm{K}), \quad a_{1}=132 \mathrm{pm} ;$ for pure Ge: $T_{m 2 \infty}=1210 \mathrm{~K}$, $\Delta_{m} S_{1 \infty}=20.57 \mathrm{~J} /(\mathrm{mol} \cdot \mathrm{K}), a_{2}=122.5 \mathrm{pm} . \Omega_{c \infty}=3500 \mathrm{~J} / \mathrm{mol}, \Omega_{s \infty}=6500 \mathrm{~J} / \mathrm{mol} \mathrm{[25].}$

Surface energy $\sigma^{L V}(x)$ of Si-Ge liquid solutions as a function of composition $x$ is obtained in [11]. The dependence is close to a linear one and can be approximated by the following relation: $\sigma^{L V}(x)=\sigma_{1}^{L V} x+\sigma_{2}^{L V}(1-x)$ where $x$ is the molar fraction of $\mathrm{Si}, \sigma_{1}^{L V}$ и $\sigma_{2}^{L V}$ are the surface energies of pure $\mathrm{Si}$ and Ge melts at the considered temperature. A similar equation is used for calculation of surface energies of Si-Ge solid solutions: $\sigma^{S V}(x)=\sigma_{1}^{S V} x+\sigma_{2}^{S V}(1-x)$. At crystal - melt interfaces, surface energies can be obtained from the simplified Girifalco-Good equation in the form which has been previously used by the authors of [29]: $\sigma^{S L}=\sigma^{S V}-\sigma^{L V}$.

Temperature dependences of surface energies for pure $\mathrm{Si}$ and $\mathrm{Ge}$ are calculated from the following linear equations (see Refs. [18,28]): $\sigma_{1}^{L V}=0.865-1.3 \cdot 10^{-4}(T-1637), \sigma_{2}^{L V}=0.587-1.05 \cdot 10^{-4}(T-1211.5)$, $\sigma_{1}^{S V}=1.081-1.3 \cdot 10^{-4}(T-1637), \sigma_{2}^{S V}=0.737-1.05 \cdot 10^{-4}(T-1211.5)$.

In the bulk state, where the surface contribution to the total energy of the system is negligible, the phase equilibria calculated by finding the minima of the Gibbs function (Eqn. 2) (see Figs. 1-3), demonstrate a good agreement with the reference data [10].

\section{Results and discussion}

In the bulk state of a material, the only, single set of compositions of co-existing liquid and solid phases at a given temperature correspond to all possible compositions of the system $(x)$. The composition of the system determines only the molar fraction of each phase directly according to the lever rule. However, the phase equilibria characteristics of nanoscale particles calculated by minimizing the Gibbs function (and plotted in Figs. 1-5) demonstrate a fundamentally different behavior in comparison with the one of the system in the bulk state. In particular, for each composition of the system $(x)$, there is a unique set of equilibrium compositions of liquid and solid phases (see Figs. 1-3 for nanoparticles containing 25, 50 and 75 at.\% Si, respectively). In each figure, the lower horizontal dashed line corresponds to the maximum temperature at which a nanoparticle of the given composition remains entirely solid ("nano-solidus"). The upper horizontal dashed line, in turn, corresponds to the minimum temperature at which a nanoparticle is still entirely liquid ("nano-liquidus"). At temperatures higher than "nano-liquidus" and lower than "nanosolidus", a minimum of the Gibbs function corresponding to a heterogeneous state does not exist.

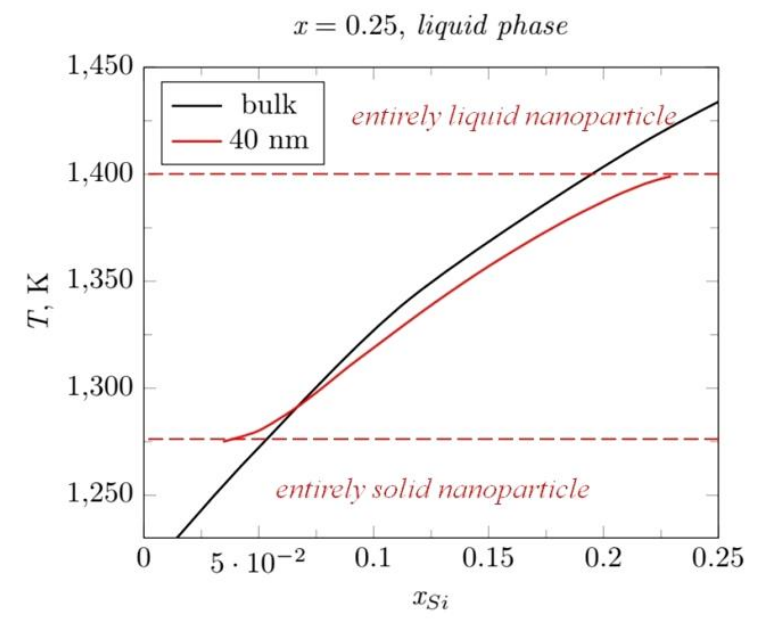

$a$

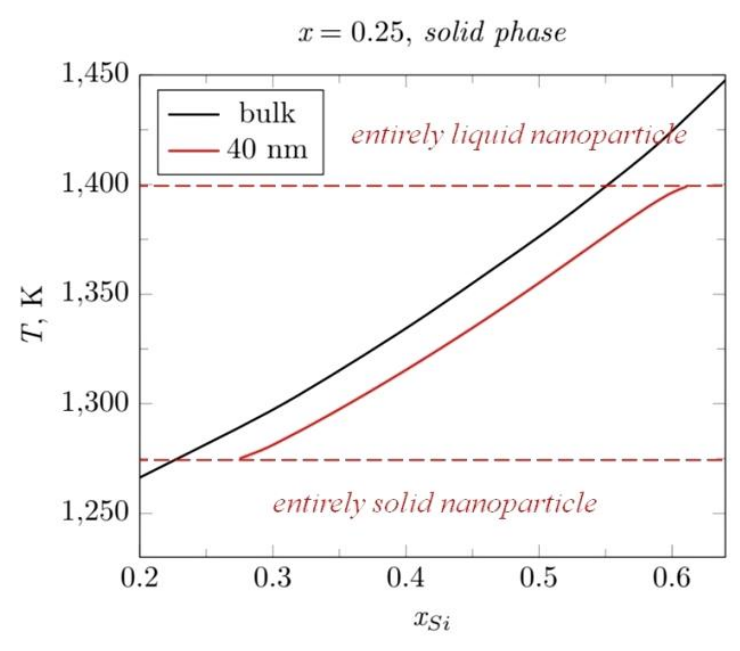

$b$

Fig.1. Temperature-dependent atomic fraction of silicon in the liquid $(a)$ and solid $(b)$ phase. The considered nanoparticle contains $25 \% \mathrm{Si}$. The red dashed lines indicate the "nano-liquidus" and "nano-solidus" temperature (the upper and lower ones, respectively). At room temperature, the particle diameter is equal to $40 \mathrm{~nm}$. The black solid line represents the bulk behaviour. 
At every composition of a nanoparticle and every temperature within the heterogeneous region, the solid phase is richer in Si than the one of the bulk system. The character of deviations of the composition of the liquid phase from the values for the bulk-state material differs depending on the temperature. At high temperatures near the "nano-liquidus", the atomic fraction of Si in a nanoparticle is higher than in the bulk state while at low temperatures near "nano-solidus", vice versa, the liquid phase is less rich in Si than such a phase in the bulk-state system. In details, this feature is illustrated in Fig. 4 where the composition of the liquid phase for equiatomic nanoparticles is plotted as a function of nanoparticle diameter at $T=1400$ (left) and $T=1500$ (right).

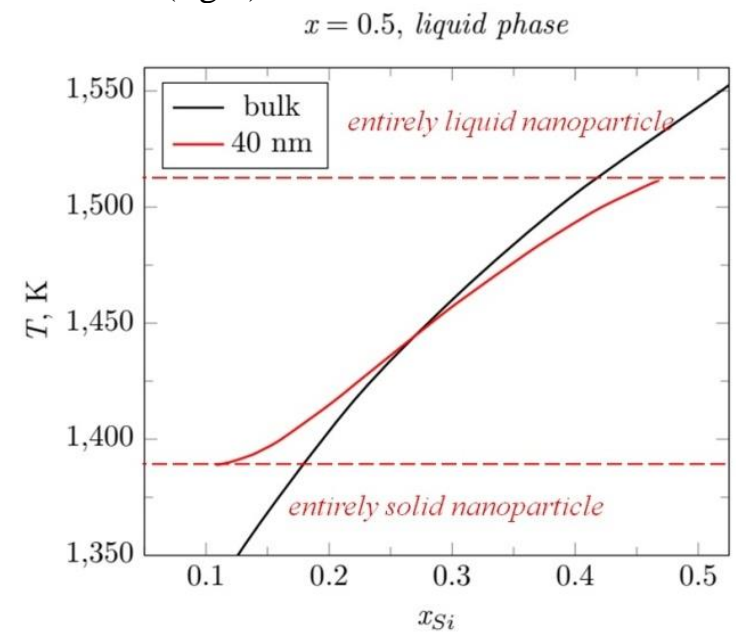

$a$

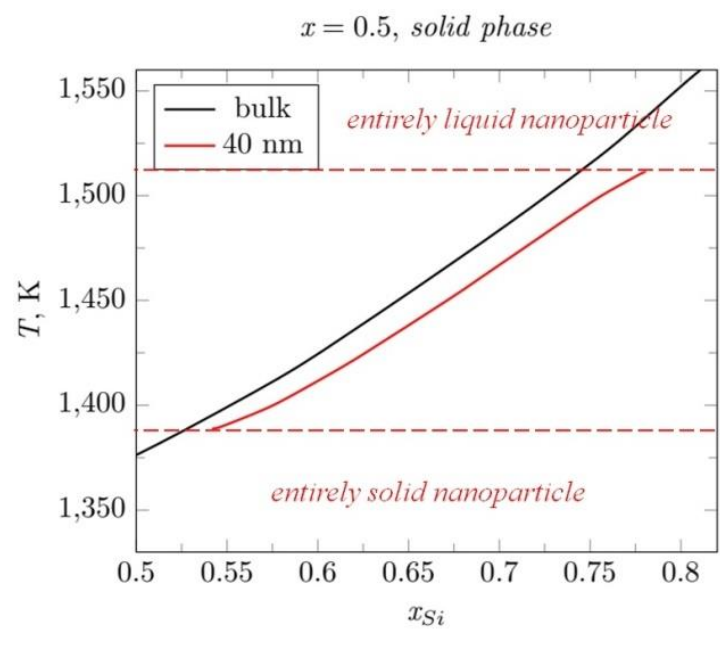

$b$

Fig. 2. Temperature-dependent atomic fraction of silicon in the liquid $(a)$ and solid $(b)$ phase. The considered nanoparticle contains 50\% Si. The red dashed lines indicate the "nano-liquidus" and "nano-solidus" temperature (the upper and lower ones, respectively). At room temperature, the particle diameter is equal to $40 \mathrm{~nm}$. The black solid line represents the bulk behaviour.
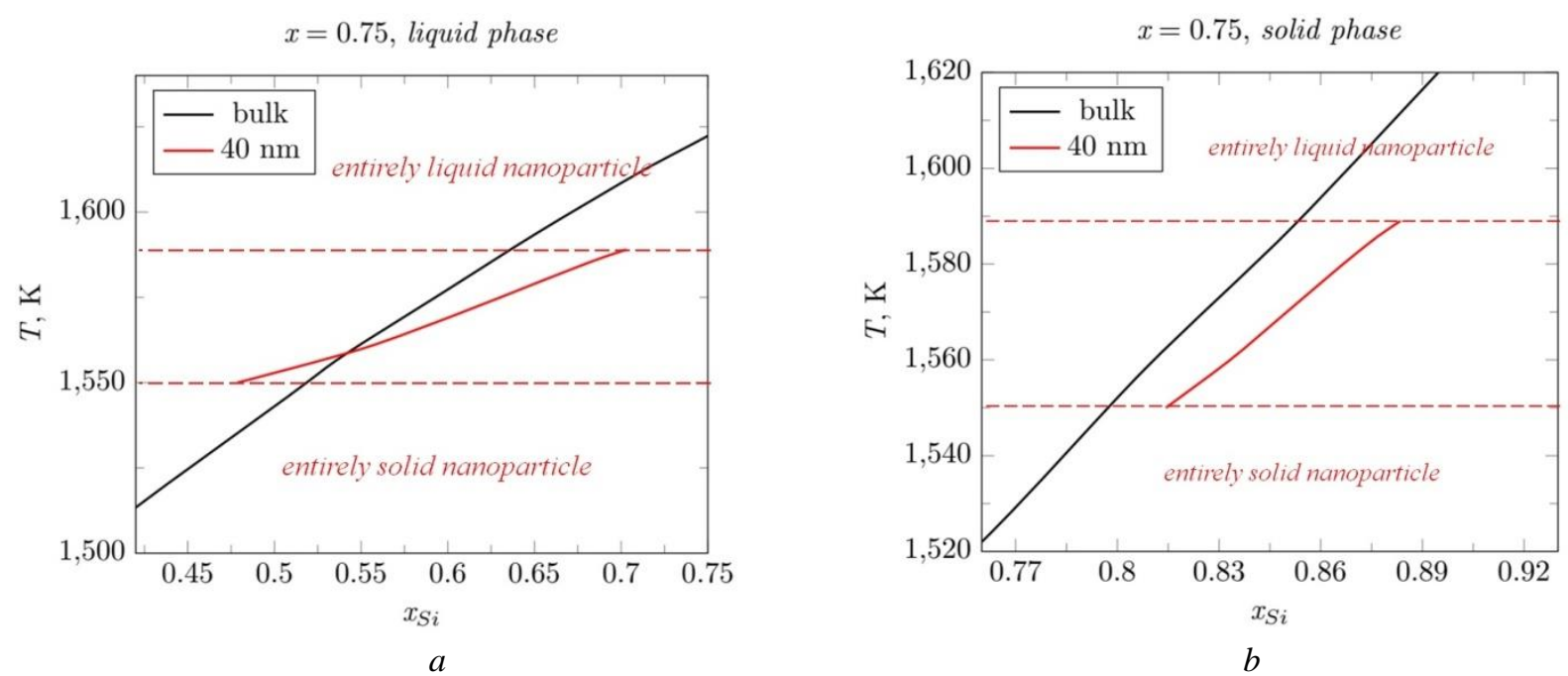

Fig. 3. Temperature-dependent atomic fraction of silicon in the liquid $(a)$ and solid $(b)$ phase. The considered nanoparticle contains $75 \% \mathrm{Si}$. The red dashed lines indicate the "nano-liquidus" and "nano-solidus" temperature (the upper and lower ones, respectively). At room temperature, the particle diameter is equal to $40 \mathrm{~nm}$. The black solid line represents the bulk behaviour.

As shown in Figs. 1-3, a decrease in the size of a particle leads to a decrease in the temperature range in which the system remains in the two-phase state. At every composition, the "nano-liquidus" of a $40-\mathrm{nm}$ diameter nanoparticle lies much below the corresponding point of the bulk liquidus line (see Fig. 5). The "nano-solidus" for all compositions considered is higher than its macroscopic analogue; the value of a temperature shift is equal to several dozens of degrees (such effect has also appeared in some previous calculations [25] where multiple simplifying presuppositions have been introduced). 

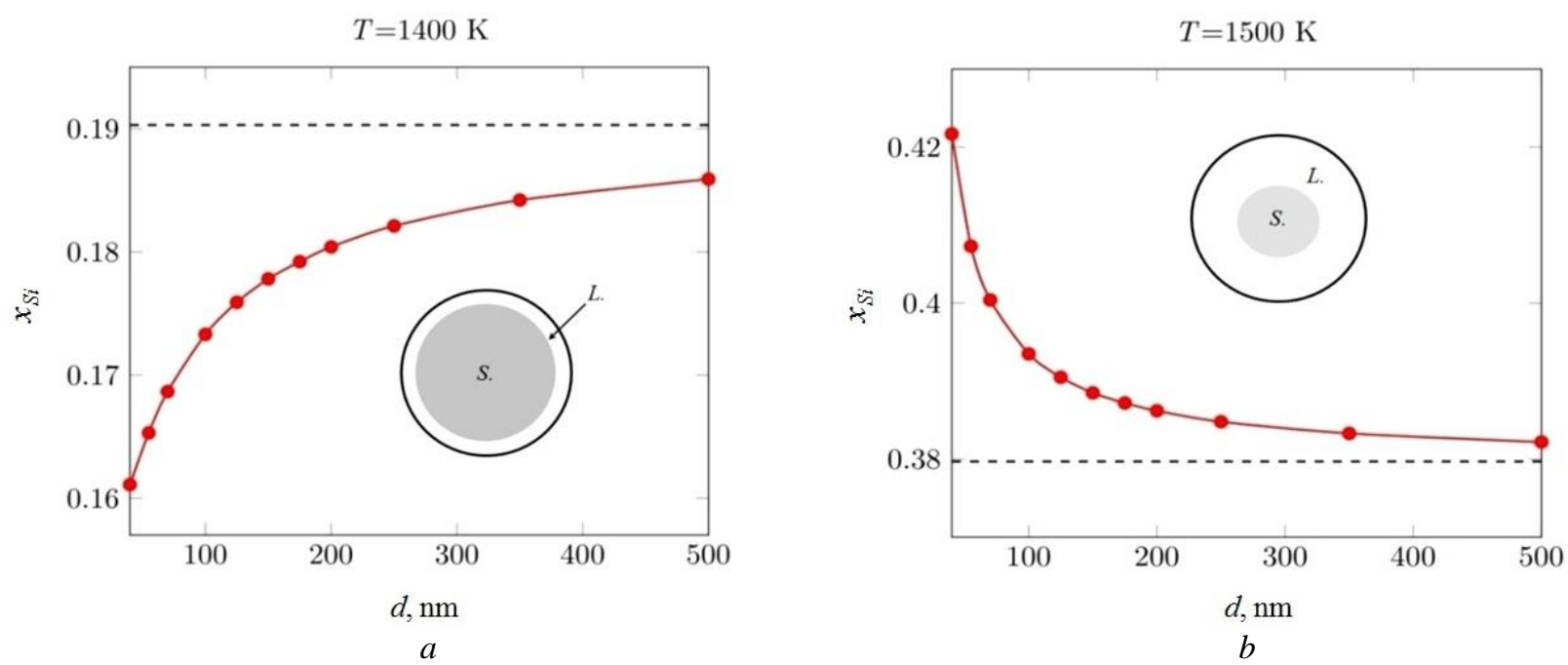

Fig. 4. Size-dependent compositions of the liquid phase (red solid lines) at $T=1400 \mathrm{~K}$ (left) and $T=1500 \mathrm{~K}$ (right) in comparison with the values for the bulk system (black dashed line).

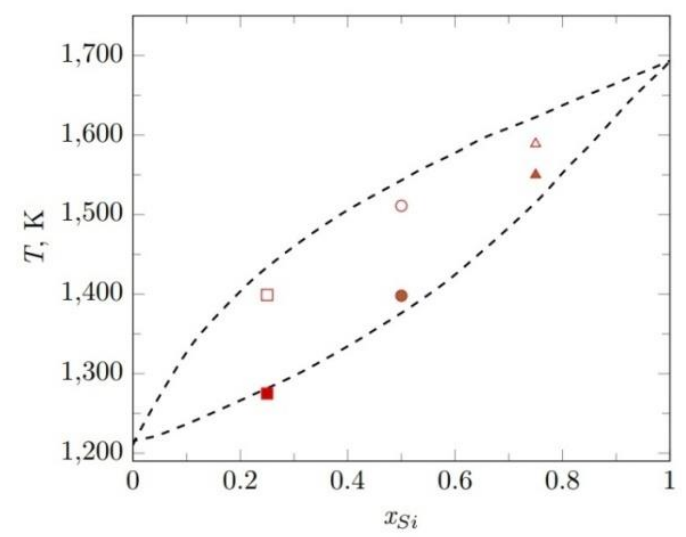

Fig. 5. "Nano-liquidus" and "nano-solidus" temperatures for nanoparticles of various compositions: 25 at. $\% \mathrm{Si}$ (squares), 50 at.\% Si (rounds), 75 at.\% Si (triangles). At room temperature, the particle diameter is equal to $40 \mathrm{~nm}$. Empty and filled markers correspond to liquidus and solidus temperatures, respectively. The phase diagram for the bulk-state material is plotted with a black dashed line.

The narrowing of the heterogeneous region for nanoparticle is caused by a great enhancement of the surface contribution to the Gibbs function of the system which makes remaining homogeneous without formation of an inner interface at temperatures near liquidus and solidus values for the bulk-state system thermodynamically more favorable. The changes in compositions of liquid and solid phases within the heterogeneous region can also be explained by the tendency to reduce the contribution of the surface energy which can possibly be realized [17,18,25,26]: (1) as a result of an increase in the total volume fraction of the melt, which is accompanied by a reduction in the inner surface area; (2) as a result of an increase in the content of the component with a higher molar volume $(\mathrm{Ge})$ in the liquid phase, which is also accompanied by a reduction in the inner surface area; (3) as a result of an increase in the content of a lower surface energy component $(\mathrm{Ge})$ in the melt, which leads to a decrease in the surface energy of the outer boundary (surface energies on the inner interface are always much lower than on the outer one [29]).

The performed simulation shows that in nanoparticles containing 50 and 75 at. \% Si at low temperatures near "nano-liquidus", all the three mechanisms are implemented. For example, in a nanoparticle containing 75 at. \% Si at $T=1555 \mathrm{~K}$, a decrease in diameter from $100 \mathrm{~nm}$ down to $40 \mathrm{~nm}$ is accompanied with an increase of the volume fraction of the liquid phase from $\sim 19$ to $\sim 23 \mathrm{vol} . \%$ and a slight increase in Ge content in the liquid phase. In an equiatomic nanoparticle of $40 \mathrm{~nm}$ in diameter at $1400 \mathrm{~K}$, there is a similar trend: the volume fraction of the melt is about $\sim 18$ vol. \% in comparison with the value of $\sim 14$ vol. \% for a $100-\mathrm{nm}$ diameter nanoparticle, and the atomic fraction of molten $\mathrm{Ge}$ in the liquid phase is higher for smaller particle than for larger ones. 
At the same time in nanoparticles with 50 and 75 at. \% Si at high temperatures near "nano-solidus" and in Ge-rich nanoparticles ( 25 at. \% Si) throughout the temperature region, mechanism 1 prevails (so the phase re-distribution is "volume-controlled"). For example, in a nanoparticle containing 75 at. \% Si at $T=1585 \mathrm{~K}$, while the diameter decreases from $100 \mathrm{~nm}$ to $40 \mathrm{~nm}$, the volume fraction of the melt dramatically increases from $\sim 46$ to $\sim 62 \mathrm{vol}$. \% and the atomic fraction of Ge in the liquid phase drops from $\sim 36$ down to 32 at. \%. In the case of an equatomic nanoparticle at $T=1500 \mathrm{~K}$, the volume fractions of the liquid phase are equal to $\sim 68$ and $\sim 76$ vol. $\%$ for 100-nm-diameter and 40-nm-diameter particles, respectively, while Ge contents in the melt are $\sim 65$ and $\sim 58$ at. \%, respectively. Finally, in a nanoparticle with 25 at. $\%$ Si at $T=1277.5 \mathrm{~K}$, the volume fraction of the liquid shell-phase grows from $\sim 9$ up to $\sim 15$ vol. \% with reducing the size from $50 \mathrm{~nm}$ to $40 \mathrm{~nm}$. At the same time, it is accompanied by a slight decrease in the atomic fraction of Ge in the liquid

phase. At $T=1390 \mathrm{~K}$, when the particle size reduces from $100 \mathrm{~nm}$ to $40 \mathrm{~nm}$, an increase in the volume fraction of the melt and a decrease in Ge content in the liquid phase also show up: from $\sim 82$ to $\sim 88$ vol. $\%$ and from $\sim 81$ to $\sim 79$ at. $\%$, respectively.

A decrease in particle diameters down to smaller values leads to more significant deviations (than the ones presented in Figs. 1-5) of equilibrium compositions and volumes of co-existing phases from the reference data for the bulk-state material. Note that for particles of a smaller size, surface tensions can become size-dependent, at very small sizes, the basic concepts of thermodynamics could hardly be applied and even the difference between solid and liquids states vanishes [30]. Modeling of such small systems requires using some other approaches (see [31], for example). Note that despite the fact the term "nanoscale effects" is widely used, there is a broad class of systems, especially polymeric ones, with great molecular volumes and masses, in which such effects manifest themselves at characteristic sizes even several thousand times higher [20,26] (and such effects should probably be called "small-amounts-of-matter effects"). In addition to this, high surface-to-volume ratios can be obtained not only in nanoparticle materials but also in mesoporous ones while the morphology of pores also influences the characteristics of phase transitions including the magnetic ones [32].

The spherical geometry of considered particles is also an idealization. A spherical particle of a given volume has the minimal surface area, taking into account more complicated morphologies [8,17-19] leads to an additional increase in the surface contribution to the total Gibbs function and some more significant shifts of characteristic points in the "phase diagram". As noted above, the shape of considered particle can be considered using one dimensionless parameter such as a coefficient of shape $[8,16,17,19]$ or a fractal dimension [8,16-19] and requires minor changes of the Eqs. above. It is necessary to mention that the configuration of the interface between co-existing phases changes during the initial stages of melting, so the introduced shape parameter needs to be temperature-dependent [18]. In the general case, the specification of phase properties of a nanosystem without detailed and precise information on its size, shape, initial composition and some other properties is almost incorrect. In description of nanoobjects, the well-known concepts such as "phase diagram", "liquidus", "solidus" obtain new meanings. The suggested model can easily be applied to the description of the melting behavior in systems of other chemical compositions including three- and polycomponent ones while the appropriate solution model and its coefficients as well as material constants (e.g. surface free energies and their temperature and composition dependence, molar volumes and their temperature dependence etc) are required for simulations. The similar effects in systems with another nature of phase transitions (e.g. nanoparticles of stratifying solid solutions, stratifying liquid solutions in small-volume droplets and pores) can also be described using a model which is quite different in mathematical formulations but shares the same thermodynamical basis (see [17,20,26] for details).

In the case of nanoparticle ensembles containing particles with different morphology characteristics, the average phase composition and functional properties depend on the size and shape distributions in an ensemble. In [33], we have suggested a method for calculating such distributions based on the combined usage of number theory (including the derivations of G.H. Hardy and S. Ramanujan on the theory of partitions), fractal geometry and statistical thermodynamics. For example, the equilibrium size distributions for nanoparticles with fractal dimension $D$ in a free-dispersed system can be expressed as follows:

$$
f_{D}\left(\phi_{p}, D, N\right): \exp \left(-\frac{\sigma A_{s p}(D)+R T \ln f_{p}}{R T}\right), f_{p}=\frac{N}{N-\phi_{p}} \exp \left\{\pi\left(\sqrt{\frac{2}{3}}\left(N-\phi_{p}\right)-\sqrt{\frac{2}{3} N}\right)\right\} .
$$


Here, $\phi_{p}=\omega\left(d_{\text {eff }} / d_{a t}\right)^{3}$ is the number of atoms in a nanoparticle, $\omega$ is the lattice packing density, $N$ is the total number of atoms in the system, $d_{\text {eff }}$ is the effective diameter of a nanoparticle (the diameter of a sphere of the same volume), $A_{s p}(D)$ is the specific surface area of the ensemble, $d_{a t}=2 r_{a t}$.

The presented estimates are in perfect accordance with the experimental data (see [33] and Refs. within) and make it possible to model the thermodynamical conditions for the realization of optimal average characteristics of nanoparticles (equilibrium compositions, phase transition temperatures, functional properties etc) as well to predict the degree at which such characteristic are "blurred" in an ensemble.

\section{Conclusion}

The performed thermodynamic analysis of phase equilibria in $\mathrm{Si}-\mathrm{Ge}$ alloy nanoparticles of various compositions leads to the following conclusions:

1) in nanoparticles, the set of phase transition temperatures (liquidus, solidus) and the compositions of co-existing phases at phase equilibrium depend on the nanoparticle size. But in addition to this, at each size and temperature, the $\mathrm{Si}$ and Ge contents in solid and liquid phases are dramatically different for different compositions of a nanoparticle. The phase equilibria in a nanosystem can hardly be described by a single phase diagram for all the composition range;

2) the character of the influence of the nanoparticle size on phase compositions differs being temperature-dependent: reducing the particle size can lead either to an increase or to a decrease in Si content in the liquid phase. The explanation of such effects is based on the existence of several mechanisms of lowering the surface energy of the system while the dominant mechanism(s) is (are) different at different compositions and temperatures.

\section{Acknowledgments}

The research has been performed in the framework of the state task for IOMC RAS.

The authors cordially thank the anonymous reviewer for the attention to this study and providing valuable remarks.

\section{REFERENCES}

1 Haddara Y.M., Ashburn P., Bagnall D.M. Silicon-germanium: properties, growth and applications. In: S. Kasap, P. Capper (eds.) Springer handbook of electronic and photonic materials, Springer handbooks. Cham, Springer, 2017, pp. 523 - 541. www.doi.org/10.1007/978-3-319-48933-9_22.

2 Vivien L., Polzer A., Marris-Morini D., et al. Zero-bias 40Gbit/s germanium waveguide photodetector on silicon. Optics express. 2012, Vol. 20, pp. 1096 - 1101. www.doi.org/10.1364/OE.20.001096.

3 Alloatti L., Srinivasan S.A., Orcutt J.S., et al. Waveguide-coupled detector in zero-change complementary metal-oxide-semiconductor. Appl. phys. lett. 2015, Vol. 107, No. 041104. www.doi.org/10.1063/1.4927393.

4 Choi J.H. High-speed devices and circuits with THz applications. Boca-Raton, CRC Press, 2017, $261 \mathrm{p}$.

5 Verma A.K., Modak P., Svane A., et al. Atomic structure and electronic properties of the $\mathrm{Si}_{x} \mathrm{Sb}_{100-x}$ phasechange memory material. Phys. rev. B. 2011, Vol. 130, No. 134205. www.doi.org/10.1103/PhysRevB.83.134205.

6 Rowe D.M. (ed.) Thermoelectric handbook macro to nano. Boca Raton, CRC Press. 2006. 1008 p.

7 Dorokhin M.V., Erofeeva I.V., Kuznetsov Yu.M., et al. Investigation of the initial stages of spark-plasma sintering of Si-Ge based thermoelectric materials. Nanosyst.: phys., chem., math. 2018, Vol. 9, pp. 622 - 630. www.doi.org/10.17586/2220-8054-2018-9-5-622-630.

8 Shishulin A.V., Fedoseev V.B., Shishulina A.V. Phonon thermal conductivity and phase equilibria of fractal Bi-Sb nanoparticles. Tech. phys. 2019, Vol. 64, No. 4, pp. 512-517. www.doi.org/10.1134/S1063784219040200.

9 Le Dantec M., Abdulstaar M., Leistner M., et al. Additive manufacturing of semiconductor silicon on silicon using direct laser melting. In: Industrializing additive manufacturing - proceedings of additive manufacturing in products and applications (AMPA-2017). 2017, pp. 104 - 116. www.doi.org/10.1007/978-3-319-66866-6_10.

10 Olesinski R.W., Abbaschian G.J. The Ge-Si (germanium-silicon) system. Bull. alloy phase diagram. 1984, Vol. 5, p. 180. www.doi.org/10.1007/BF02868957.

11 Ricci E., Amore S., Giuranno D., et al. Surface tension and density of Si-Ge melts. J. chem. phys. 2014, Vol. 140, No. 214704. www.doi.org/10.1063/1.4879775.

12 Wang Q., Chang J., Wang H.P. Thermodynamical properties and atomic structure of liquid Si-Ge alloys. Mater. chem. phys. 2019, Vol. 221, pp. 224 - 231. www.doi.org/10.1016/j.matchemphys.2018.09.043.

13 Liang L.H., Liu D., Jiang Q. Size-dependent continuous binary solution phase diagram. Nanotechnology. 2003, Vol. 14, pp. 438 - 442. www.doi.org/10.1088/0957-4484/14/4/306. 
14 Bajaj S., Haverty M.G., Arróyave R., et al. Phase stability in nanoscale material systems: extensions from bulk phase diagrams. Nanoscale. 2015, Vol. 7, No. 9868. www.doi.org/10.1039/C5NR01535A.

15 Bonham B., Guisbiers G. Thermal stability and optical properties of Si-Ge nanoparticles. Nanotechnology. 2019, Vol. 28, No. 245702. www.doi.org/10.1088/1361-6528/aa726b.

16 Fedoseev V.B., Potapov A.A., Shishulin A.V., Fedoseeva E.N. Size and shape effect on the phase transitions in a small system with fractal interphase boundaries. Eurasian phys. tech. j. 2017, Vol. 14, No.1, pp. 18 - 24.

17 Shishulin A.V., Fedoseev V.B. On some peculiarities of stratification of liquid solutions within pores of fractal shape. J. mol. liq. 2019, Vol. 278, pp. 363 - 367. https://doi.org/10.1016/j.molliq.2019.01.050.

18 Shishulin A.V., Fedoseev V.B., Shishulina A.V. Melting behavior of fractal-shaped nanoparticles (the example of Si-Ge system). Tech. phys. 2019, Vol. 64, No. 9, pp. 1343 - 1349. https://doi.org/10.1134/S1063784219090172.

19 Shishulin A.V., Potapov A.A., Fedoseev V.B. Phase equilibria in fractal core-shell nanoparticles of $\mathrm{Pb}_{5}\left(\mathrm{VO}_{4}\right)_{3} \mathrm{Cl}-\mathrm{Pb}_{5}\left(\mathrm{PO}_{4}\right)_{3} \mathrm{Cl}$ system: the influence of size and shape. Advances in artificial systems for medicine and education II. Cham., Springer. 2020, pp. 405 - 413. https://doi.org/10.1007/978-3-030-12082-5_37.

20 Shishulin A.V., Fedoseev V.B. Thermal stability and phase composition of stratifying polymer solutions in small-volume droplets. J. eng. phys. thermophys. 2020, Vol. 93, No. 4, pp. 802 - 809. www.doi.org/10.1007/s10891020-02182-9.

21 Mendoza-Pérez R., Muhl S. Phase diagrams of refractory bimetallic nanoalloys. J. nanopart. res. 2020, Vol. 22, No. 36. www.doi.org/10.1007/s11051-020-05035-x.

22 Geoffrion L.-D., Guisbiers G. Chemical ordering in $\mathrm{Bi}_{1-x} \mathrm{Sb}_{x}$ nanostructures: alloy, janus or core-shell. J. phys. chem. C. 2020, Vol. 124, No. 25, pp. 14061 - 14068. www.doi.org/10.1021/acs.jpcc.0c04356.

23 Shishulin A.V., Fedoseev V.B., Shishulina A.V. Environment-dependent phase equilibria in a small-volume system in the case of decomposition of Bi-Sb solid solutions. Butlerov commun. 2017. Vol. 51, No. 7 , pp. 31 - 37 [in Russian].

24 Straumal B., Baretzky B., Mazilkin A., et al. Increase of Mn solubility with decreasing grain size in ZnO. J.Eur. ceram. soc. 2009, Vol. 29, pp. 1963 - 1970. www.doi.org/10.1016/j.jeurceramsoc.2009.01.005.

25 Shishulin A.V., Fedoseev V.B. Effect of initial composition on the liquid-solid phase transition in Cr-W alloy nanoparticles. Inorg. mater. 2019, Vol. 55, no. 1, pp. 14 - 18. www.doi.org/10.1134/S0020168519010138.

26 Shishulin A.V., Fedoseev V.B. Features of the influence of the initial composition of organic stratifying mixtures in microsized pores on the mutual solubility of components. Tech. phys. lett. 2020. Vol. 46, No. 9, pp. $938-$ 941. www.doi.org/10.1134/S1063785020090291.

27 Potapov A.A. On the issues of fractal radio electronics: Processing of multidimensional signals, radiolocation, nanotechnology, radio engineering elements and sensors. Eurasian phys. tech. j. 2018, Vol. 15, No. 2, pp. 5 - 15.

28 Hourlier D., Perrot P. Au-Si and Au-Ge phase diagrams for nanosystems. Mater. sci. forum. 2010, Vol. 653, pp. 77 - 85. www.doi.org/10.4028/www.scientific.net/MSF.653.77.

29 Alymov M.I., Shorshorov N.Kh. Effect of size factors on the melting point and surface tension of ultrafine particles. Metally. 1999, Vol. 2, pp. 29 - 31 [in Russian].

30 Magomedov M.N. On the minimum size of a nanoparticle when the difference between solid and liquid states disappears. Herald of the Bauman Moscow state technical university. 2012, Vol. 44, pp. 36 - 49 [in Russian].

31 Magnin Y., Zappelli A., Amara H., et al. Size-dependent phase diagrams of nickel-carbon nanoparticles. Phys. rev. lett. 2015, Vol. 115, No. 205502. www.doi.org/10.1103/PhysRevLett.115.205502.

32 Shishulin A.V., Potapov A.A., Shishulina A.V. On the transition between ferromagnetic and paramagnetic states in mesoporous materials with fractal morphology. Eurasian phys. tech. j. 2021, Vol. 18, No.2, pp. 6 - 11.

33 Fedoseev V.B., Shishulin A.V. On the size distribution of dispersed fractal particles. Tech. phys. 2021, Vol. 66, No. 1, pp. 34 - 40. https://doi.org/10.1134/S1063784221010072. 